\title{
DISEÑO DE UN ÍNDICE DE PELIGRO DE INCENDIO FORESTAL PARA LA PROVINCIA PINAR DEL RÍO, CUBA
}

\author{
Yulian Carrasco Rodríguez ${ }^{1}$, Marcos Pedro Ramos Rodríguez ${ }^{2}$, Antonio Carlos Batista ${ }^{3}$, \\ Luis Wilfredo Martínez Becerra ${ }^{4}$, Alexandre França Tetto ${ }^{3}$ \\ ${ }^{1}$ Universidad de Pinar Del Rio, Departamento Forestal, Pinar del Río, Cuba - yulianc@upr.edu.cu \\ ${ }^{2}$ Universidad Estatal Del Sur de Manabí, Manabí, Ecuador - marcos.ramos@unesum.edu.ec \\ ${ }^{3}$ Universidade Federal do Paraná, Departamento de Ciencias Forestales, Curitiba, Paraná, Brasil - batistaufpr@ufpr.br; tetto@ufpr.br \\ ${ }^{4}$ Universidad de Pinar Del Rio, Facultad Forestal y Agronomía, Pinar del Río, Cuba - wmartinez@upr.edu.cu \\ Recebido para publicação: 11/07/2016 - Aceito para publicação: 03/11/2016
}

\begin{abstract}
Resumen
La dinámica de un incendio forestal está condicionada tanto por los factores permanentes como por los variables, siendo estos los que determinan a corto plazo la ocurrencia o no de los incendios. Una de las herramientas utilizadas para la evaluación del peligro de incendio son los índices de peligro. La provincia Pinar del Río fue en Cuba la primera en ocurrencia de incendio y la segunda en áreas quemas en los últimos 8 años. El objetivo de esta investigación fue diseñar un índice de peligro de incendios forestales para la provincia Pinar del Río. Mediante el análisis de las correlaciones entre las variables meteorológicas y la ocurrencia de incendio se determinaron las variables que más se relacionan con estas ocurrencias. Posteriormente, mediante el método de regresión logística stepwise de Wald, se determinaron todos los posibles modelos que pudieran explicar la ocurrencia de incendios. El mejor punto de corte fue definido según el Índice de Youden. El mejor modelo se seleccionó considerando la bondad de ajuste de Hosmer-Lemeshow, la capacidad predictiva, el estadístico de $2 L L$, el coeficiente de Nagelkerke, y la curva COR. Los resultados indicaron que la humedad relativa y la velocidad del viento fueron las variables que mejor explicaron la ocurrencia de incendios. Utilizando estos resultados se diseñó el modelo que mejor explica la relación entre las variables analizadas, obteniéndose así el índice Fórmula de Pinar del Río (FPR) para la provincia Pinar del Río.

Palabras-clave: Peligro de incendio; correlación; regresión logística; índice FPR.
\end{abstract}

\section{Resumo}

Proposta de um índice de perigo de incêndios para a província de Pinar del Río, Cuba. A dinâmica de um incêndio está condicionado tanto por fatores permanentes como variáveis, sendo esses últimos os que determinam a ocorrência ou não ocorrência de incêndios a curto prazo. Uma das ferramentas utilizadas para a avaliação do perigo de incêndio são os índices de perigo. A província de Pinar del Río foi a primeira em ocorrência de incêndios e a segunda em áreas queimadas nos últimos 8 anos em Cuba. O objetivo desta pesquisa foi propor um índice de perigo de incêndio florestal para a província Pinar del Río. Ao analisar as correlações entre variáveis meteorológicas e a ocorrência de incêndios foram determinadas as variáveis que estão relacionadas com estas ocorrências. Posteriormente, mediante o método de regressão logística stepwise de Wald, foram determinados todos os modelos possíveis que poderiam explicar a ocorrência de incêndios. $\mathrm{O}$ melhor modelo foi escolhido considerando a qualidade do ajuste de Hosmer-Lemeshow, a capacidade preditiva, $-2 L L$ estatística, o coeficiente de Nagelkerke e a curva ROC. Os resultados indicaram que a umidade relativa e velocidade do vento foram as variáveis que melhor explicaram a ocorrência de incêndio. Usando estes resultados foi desenvolvido o modelo que melhor explica a relação entre as variáveis analisadas, obtendo-se assim o índice Fórmula de Pinar del Río (FPR) para a província de Pinar del Río.

Palavras-chave: Perigo de incêndio; correlações; regressão logística; índice FPR.

\section{INTRODUCCIÓN}

Los bosques son parte integrante de los sistemas sustentadores de la vida en la Tierra y desempeñan un importante papel en la regulación de la atmósfera y el clima. Según Organización para la Agricultura y la Alimentación (FAO) (2015), el 30,6 por ciento de la superficie del planeta está cubierta por bosques, ocupando un área aproximada de 3,999 millones de hectáreas, la deforestación continúa a un ritmo alarmante, estimándose una pérdida neta anual de 13 millones de hectáreas de bosques durante el último decenio, siendo una de las causas los incendios forestales, estimándose una superficie quemada media anual de 27843 hectáreas. 
En Cuba, al igual que en el resto del mundo, el fuego ha sido y es utilizado por los agricultores como una herramienta eficaz y económica para eliminar residuos de cosechas, limpiar terrenos y renovar los pastos. Si bien los fuegos forman parte de las características normales de varios ecosistemas en el mundo, resulta uno de los principales siniestros que atentan contra el desarrollo de las áreas forestales (DOMÍNGUEZ et al., 2008).

Según el Consejo de Defensa Nacional (CDN) en el año 2010 el principal riesgo de incendios se localiza en áreas forestales, siendo Pinar del Río una de las provincias donde históricamente se registran los mayores números de incendios forestales en el país.

Acorde con los datos ofrecidos por la Oficina Nacional de Estadística e Información (ONEI), durante los años (2007-2014), han ocurrido en Cuba 3129 incendios que han afectado una superficie de 59 366,56 ha. De estos totales corresponden a la provincia de Pinar del Río 606 incendios $(19,40 \%)$ y 9591,88 hectáreas afectadas $(16,20 \%)$ siendo la primera provincia en cuanto a incendios ocurridos y la segunda en áreas afectadas. Esta situación indica la importancia que tiene tratar de reducir, tanto el número de incendios, como las superficies por ellos afectadas ONEI (2014).

Una actividad importante que puede contribuir significativamente al logro de este propósito puede ser el diseño de un índice de peligro de incendios bien ajustado al área de estudio, lo que repercutiría en la mejora de la toma de decisiones para las actividades básicas del manejo del fuego: uso, prevención y extinción del fuego.

Los índices de peligro de incendio son números que reflejan, anticipadamente, la probabilidad de ocurrencia de un incendio, así como la facilidad del mismo de propagarse, con base en las condiciones atmosféricas del día o de una secuencia de días (VÉLEZ, 2009).

En correspondencia con lo anterior, Soares et al. (2009), plantean que dentro de las medidas preventivas existentes, la utilización de un índice de peligro de incendio forestal confiable es factor fundamental para una planificación más eficiente de las medidas de prevención y para la adopción de acciones rápidas y efectivas en las actividades de combate, buscando la reducción de las pérdidas, con la disminución de las áreas quemadas, así como los prejuicios financieros y ambientales derivados de la ocurrencia de tales eventos catastróficos.

Independientemente de lo anterior, según Nunes et al. (2010), la utilización de un índice de peligro desajustado puede llevar a tomar malas decisiones con respecto a los procedimientos para la prevención y combate contra los incendios forestales.

En correspondencia con lo anterior el objetivo de este trabajo fue diseñar una fórmula para la determinación del índice de peligro de incendios forestales en la provincia Pinar del Río, probándose la hipótesis de que la utilización de métodos estadísticos como correlación y regresión logística entre los incendios forestales y las variables meteorológicas en la provincia Pinar del Río, permite el diseño de un índice debidamente ajustado para la zona de estudio.

\section{MATERIALES Y MÉTODOS}

\section{Caracterización del área de estudio}

La investigación fue desarrollada en la provincia Pinar del Río, la más occidental de la isla de Cuba, la cual tiene una superficie total de $8883,74 \mathrm{~km}^{2}$ (888 374 ha) clasificándose como la tercera más grande de Cuba. La misma está ubicada entre los $21^{\circ} 52^{\prime}$ de latitud norte y los $84^{\circ} 57^{\prime}$ de longitud oeste según la Comisión Nacional de Nombres Geográficos (2000) citado por ONEI (2014).

Según Köppen (1936) citado por Wadsworth (2000), el clima en Pinar del Río es Aw (clima tropical, con mes más frío, con temperatura mayor de $18{ }^{\circ} \mathrm{C}$ y una estación seca con al menos un mes con menos de $600 \mathrm{~mm}$ ). La precipitación media anual es de $1589,64 \mathrm{~mm}$, la temperatura media anual de $25,10^{\circ} \mathrm{C}$ y la humedad relativa media anual de $76,23 \%$.

\section{Obtención de los datos de ocurrencia de incendios y meteorológicos}

Los datos sobre ocurrencias de incendios fueron obtenidos en la Oficina del Cuerpo de Guardabosques en Pinar del Río. Todos los datos corresponden al período comprendido entre el 01/01/1994 y el 31/12/2013, totalizando 20 años de observaciones.

La serie de datos meteorológicos utilizados para el trabajo fue obtenida de la base de datos del Centro Meteorológico Provincial de Pinar del Río, perteneciente al Instituto de Meteorología de la República de Cuba (INSMET). Las variables consideradas fueron humedad relativa, temperatura del aire, temperatura del punto de rocío, velocidad del viento y precipitación. Todos los valores fueron medidos diariamente a las 13:00 horas en cada una de las seis Estaciones Meteorológicas seleccionadas en la provincia.

A partir de las variables disponibles y utilizando el Editor de Visual Basic el cual es una herramienta de entorno combinado de programación integrado a Microsoft Excel y desarrollando bucles, se obtuvieron dos nuevas variables, consideradas importantes para el análisis. Estas variables fueron el número de días sin lluvias 
antes de la ocurrencia del incendio y el número de días sin lluvia mayor o igual a $10 \mathrm{~mm}$ antes de la ocurrencia del incendio.

\section{Proceso metodológico}

Para el diseño del índice de peligro de incendios forestales se analizaron las relaciones entre ocurrencias de incendios y variables meteorológicas. La relación entre las variables se determinó a través del coeficiente de correlación no paramétrica de Spearman, considerando una probabilidad de significación del 5\%. La interpretación de los valores de la correlación fue hecha de acuerdo con la propuesta de Salinas (2007), la cual se muestra en la tabla 1 .

Tabla 1. Interpretación de los valores de las correlaciones de Spearman.

Tabela 1. Interpretação dos valores de correlação de Spearman.

\begin{tabular}{ll}
\hline Valor de $\mathbf{r}$ & Interpretación \\
\hline 0,00 & Nula \\
$0,00-0,19$ & Muy baja \\
$0,20-0,39$ & Baja \\
$0,40-0,59$ & Moderada \\
$0,60-0,79$ & Alta \\
$0,80-1,00$ & Muy alta \\
1,00 & Perfecta \\
\hline
\end{tabular}

También se determinaron modelos predictivos considerando a los incendios registrados como variable dependiente. Debido a la complejidad en la distribución de los mismos y su relación con el resto de los factores que propician el desarrollo de un incendio, se procedió a la transformación de la misma en una variable dicotómica binaria, nombrándose ocurrencia de incendios, donde considerando la presencia o ausencia de los mismos, toma valores de 1 y 0.

La selección de las variables que integraron los diferentes modelos, se realizó utilizando la regresión logística binaria en su método stepwise de Wald. Para la determinación del punto de corte óptimo se utilizó la metodología propuesta por Fuentes (2013), utilizando el Índice de Youden (IY) el cual establece una solución donde se optimiza el valor de los estadígrafos sensibilidad (S) y especificidad (E): IY= ((S+E)/100)-1. De modo que se elige como punto de corte óptimo aquel que minimizando la suma de los errores (Falsos Positivos (FP)+Falsos Negativos (FN)) maximice el Índice de Youden utilizando siempre un intervalo de confianza del 95\%.

El modelo de regresión logística se define en la ecuación 1:

$$
\begin{aligned}
& P_{i}=\frac{1}{1+e^{-z}} \\
& Z=\beta_{0}+\beta_{1} X_{1}+\beta_{2} X_{2}+\ldots+\beta_{p} X_{p}
\end{aligned}
$$

Donde $P i$ es la probabilidad de ocurrencia de un suceso, z se define en la ecuación 2 como la combinación de variables independientes con sus coeficientes de regresión $(\beta), \mathrm{X}$ el valor de cada variable independiente y $e$ la base del logaritmo natural (AFIFI et al., 1990 y McGREW et al., 1993 citados MARTINEZ et al., 2009).

Entre las posibilidades de modelos de regresión logística binaria se aplica el modelo logit: $\log \left(\frac{\mathrm{p}}{1-\mathrm{p}}\right)=\mathrm{x}^{\mathrm{T}} \boldsymbol{\beta}$. Siendo $\boldsymbol{x}^{\boldsymbol{T}}$ el vector de las variables explicativas y $\boldsymbol{\beta}$ el vector de los parámetros (SYPHARD et al., 2008). El término a la derecha de la igualdad es la expresión de una recta, idéntica a la del modelo general de regresión lineal. Siendo la ecuación inicial del modelo de tipo exponencial, permite su transformación logarítmica (logit) lo que posibilita su uso como una función lineal. ecuación 3.

Se define entonces la transformación (logit) como el logaritmo natural (de base " $e$ ") obteniéndose la

$$
\operatorname{Ln}\left(\frac{\mathrm{p}}{1-\mathrm{p}}\right)=\operatorname{Ln}\left(e^{\beta_{0}+\beta_{\mathrm{i}} \mathrm{x}}\right)=\beta_{0}+\beta_{1} \mathrm{x}_{1}+\beta_{2} \mathrm{x}_{2} \ldots+\beta_{\mathrm{n}} \mathrm{x}_{\mathrm{n}}
$$

Como se observa en la ecuación 3, la transformación (logit) es lineal en los parámetros del modelo, de manera que permite realizar análisis muy similares a los de la regresión lineal. Un coeficiente positivo aumenta la probabilidad de ocurrencia del evento, en tanto que uno con signo negativo la disminuye. Con el interés de

FLORESTA, Curitiba, PR, v. 47, n. 1, p. 65- 74, jan. / mar. 2017.

Rodríguez, Y. C. et al.

ISSN eletrônico 1982-4688

DOI: $10.5380 /$ rf.v47i1.47652 
obtener la relación entre las variables dependiente e independiente solo se utilizará el valor de Z en función de la ocurrencia de incendio.

La selección del mejor modelo entre todos los posibles no se hizo en base a un único criterio, sino que se analizaron los criterios de bondad de ajuste de Hosmer-Lemeshow, capacidad predictiva, el estadístico de 2LL (-2 Logaritmo de Likelihood = -2 veces el logaritmo de la verosimilitud), coeficiente de Nagelkerke, así como la representación gráfica de la curva COR (en inglés ROC, del acrónimo de Receiver Operating Characteristic). Además, se procuró encontrar modelos poco complejos tanto en número de variables como en facilidad de obtención de las variables, es decir, modelos fáciles de instrumentar para su aplicación operativa.

Después de la determinación de las ecuaciones de regresión logística resultantes para todas las combinaciones obtenidas mediante el método stepwise de Wald, se procedió al cálculo de la probabilidad que ofrece cada uno de los modelos a proponer, seleccionando de esta forma el que mayor porcentaje de probabilidad y de skill score presente.

Con basamento en la revisión de literatura y en la analogía con otras escalas de peligro de incendio en el mundo como las de los índices Nesterov, Formula de Monte Alegre (FMA) y Formula de Monte Alegre Modificada $\left(\mathrm{FMA}^{+}\right)$, se decidió a utilizar una escala con cinco grados de peligro tomándose en cuenta los valores máximos y mínimos del modelo evaluado y los resultados de los índices obtenidos diariamente. La determinación de la escala de peligro se realizó utilizando como criterio las premisas sobre las distribuciones que indican el ajuste de los índices, planteadas por Nunes et al. (2010), donde argumentan que el número de días previstos en cada clase de peligro debe tener una relación inversa con la clase de peligro, de modo que cuanto mayor sea la clase de peligro, menor es el número de días previstos para ella, también se tuvo en cuenta la planteada por Tetto et al. (2010), exponiendo que una distribución deseable para esta variable es también cuando la mayor cantidad de días previstos son agrupados en la clase tres (Media) disminuyendo gradualmente para las clases uno (Nulo) y cinco (Muy alto).

Según Secundo et al. (2015), los índices de peligro basados en variables meteorológicas como el de Nesterov (1965), Telicyn (1971), Soares (1972), Rodríguez y Moretti (1988) y Nunes (2005), tienen como característica común su carácter acumulativo, los cuales utilizan la precipitación como variable de restricción. Estas restricciones indican que el índice determinado por la fórmula se suma diariamente hasta que ocurra una lluvia. La cantidad de precipitación determina entonces las modificaciones a efectuar en los valores del índice, desde una reducción parcial del mismo hasta el abandono total del valor acumulado. En correspondencia con esto, el índice diseñado es también de carácter acumulativo y utiliza las mismas restricciones propuestas por Nunes et al. (2006), donde será modificado el porcentaje partiendo de la acumulación de precipitación mayor o igual a $13 \mathrm{~mm}$, quedando expresado según se muestra en la tabla 2.

Tabla 2. Restricciones a la sumatoria de la FPR de acuerdo a la precipitación del día.

Tabela 2. Restrições à soma do FPR de acordo com a precipitação do dia.

\begin{tabular}{cl}
\hline Lluvia del día $(\mathbf{m m})$ & Modificación en el cálculo \\
\hline$\leq 2,4$ & Ninguna \\
$2,5-4,9$ & $\begin{array}{l}\text { Disminuir un } 30 \% \text { al valor del FPR acumulado y sumar el }[13,608- \\
(0,276 * \mathrm{~V})-(0,182 * \mathrm{Hr})] \text { calculado para el día }\end{array}$ \\
$5,0-9,9$ & $\begin{array}{l}\text { Disminuir un } 60 \% \text { al valor del FPR acumulado y sumar el }[13,608- \\
(0,276 * \mathrm{~V})-(0,182 * \mathrm{Hr})] \text { calculado para el día }\end{array}$ \\
& $\begin{array}{l}\text { Disminuir un } 80 \% \text { al valor del FPR acumulado y sumar el }[13,608- \\
(0,276 * \mathrm{~V})-(0,182 * \mathrm{Hr})] \text { calculado para el día }\end{array}$ \\
& Interrumpir el cálculo $(\mathrm{FPR}=0)$ e iniciar la sumatoria el día \\
& siguiente \\
\hline
\end{tabular}

\section{RESULTADOS Y DISCUSIÓN}

\section{Relaciones entre ocurrencias de incendios y variables meteorológicas}

En la tabla 3 se muestran los valores de la correlación existente entre la ocurrencia de incendio y las variables meteorológicas. Puede observarse que en todos los casos existe relación entre las variables $(\mathrm{p} \leq 0,05)$. No obstante, las correlaciones son muy bajas, excepto para el caso de la ocurrencia de incendios y la humedad relativa, que es baja. 
Tabla 3. Correlación entre la ocurrencia de incendios y las variables meteorológicas en la provincia Pinar del Río (1994-2013).

Tabela 3. Correlação entre a ocorrência de incêndios e as variáveis meteorológicas na província de Pinar del Río (1994-2013).

\begin{tabular}{lrl}
\hline Variables & \multicolumn{1}{c}{ r } & p \\
\hline Ocurrencia de incendios - Precipitación & $-0,085$ & 0,000 \\
Ocurrencia de incendios - Temperatura del aire & 0,105 & 0,000 \\
Ocurrencia de incendios - Temperatura punto de rocío & $-0,093$ & 0,000 \\
Ocurrencia de incendios - Humedad relativa & $-0,220$ & 0,000 \\
Ocurrencia de incendios - Velocidad del viento & 0,082 & 0,000 \\
Ocurrencia de incendios - NúmDías_SLL & 0,060 & 0,000 \\
Ocurrencia de incendios - NúmDías_SLL $\geq 10 \mathrm{~mm}$ & 0,105 & 0,000 \\
\hline
\end{tabular}

Se puede subrayar que la precipitación y la ocurrencia de incendios a pesar de tener una correlación muy baja, presentan una relación inversa muy proporcional. La distribución de estas variables a través de los meses del año se observa en la figura 1. Puede observarse el comportamiento inverso de ambas variables.

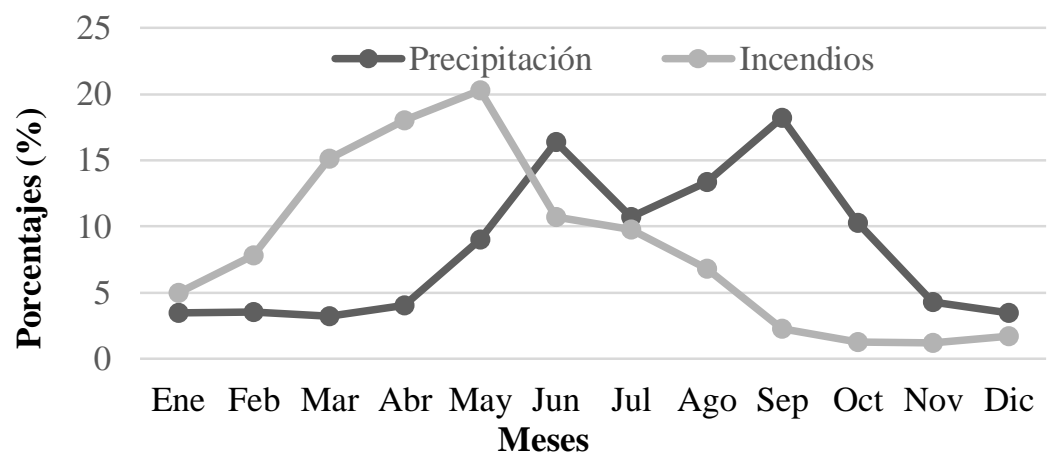

Figura 1. Porcentajes de precipitación y ocurrencia de incendios en la provincia Pinar del Río (1994-2013).

Figura 1. Porcentagem de precipitação e incêndios florestais na província Pinar del Río (1994-2013).

Con el objetivo de buscar un modelo eficiente que permita realizar un pronóstico acertado de las probabilidades de ocurrencia de incendio, se evaluó la posibilidad de valorar todos los tipos de modelos que manifestaron una relación entre las variables analizadas.

Una de las condiciones de la regresión logística es la no existencia de la multicolinealidad entre las variables que forman parte del modelo de regresión (Yang et al., 2008). Según Hernández et al. (2007) la no multicolinealidad se puede demostrar mediante los valores de los coeficientes de correlación de Spearman, demostrándose de esta forma con las bajas relaciones mostradas en la tabla 3.

Se aplicó la regresión logística obteniéndose como resultado tres posibles modelos a validar. En la tabla 4 se muestra que los modelos globalmente son significativos, evaluados con la prueba del logaritmo del cociente de verosimilitudes, que en el SPSS se denomina "prueba de ómnibus", obtenidos por la regresión logística, indicando que los tres posibles modelos tienen una significación menor que 0,05 , lo que demuestra que las variables independientes que componen esos posibles modelos explican el comportamiento de la ocurrencia de incendios.

Tabla 4. Ajuste de los modelos logísticos obtenidos.

Tabela 4. Ajuste dos modelos logísticos obtidos.

\begin{tabular}{cccc}
\hline MRL & Chi-cuadrado & gl. & Sig. \\
\hline Modelo 1 & 26,114 & 1 & 0,000 \\
Modelo 2 & 51,705 & 2 & 0,000 \\
Modelo 3 & 55,326 & 3 & 0,000 \\
\hline
\end{tabular}

MRL (Modelo de Regresión Logística)

En la tabla 5 se muestra los valores obtenidos para cada modelo de -2 veces el logaritmo de la verosimilitud (-2LL) y el coeficiente de Nagelkerke. Obsérvese que hay una disminución del -2LL, en relación al primer modelo, correspondiendo el menor al tercero. Mientras menor es -2LL, mayor es la verosimilitud y mejor el 
ajuste del modelo. El coeficiente de determinación $\mathrm{R}^{2}$ de Nagelkerke aumenta desde el primero hasta el tercer modelo teniendo el modelo dos y tres los valores 0,20 y 0,21 respectivamente, confirmándose que el 20 y el $21 \%$ de la varianza es explicada por las variables predictora introducidas en el modelo dos y tres, según corresponde.

Tabla 5. Ecuaciones de los modelos de regresión logísticos.

Tabela 5. Equações de modelos de regressão logística.

\begin{tabular}{llcc}
\hline MRL & Ecuación & -2LL & Coeficiente de Nagelkerke $\left(\boldsymbol{R}^{2}\right)$ \\
\hline 1 & $\mathrm{Z}=7,029-0,115 * \mathrm{Hr}$ & 293 & 0,102 \\
2 & $\mathrm{Z}=13,608-0,276 * \mathrm{~V}-0,182 * \mathrm{Hr}$ & 268 & 0,200 \\
3 & $\mathrm{Z}=13,107-0,276 * \mathrm{~V}-0,178 * \mathrm{Hr}+0,156 *$ NúmDías_SLL & 264 & 0,211 \\
\hline \multicolumn{2}{l}{ Logaritmo de la verosimilitud -2 (-2LL) MRL (Modelo de regresión logístico) }
\end{tabular}

Los resultados de la prueba de Hosmer y Lemeshow para cada modelo, basado en la comparación entre los casos observados y los casos pronosticados se muestran en la tabla 6. Puede observarse que para los tres modelos el Chi cuadrado no es significativo, aumentando su valor desde el primero hasta el tercer modelo, lo cual indica que no hay motivos para pensar que los resultados predichos sean diferentes de los observados (o que si hay diferencias pueden explicarse razonablemente por el azar o error del muestreo) y que los modelos pueden considerarse aceptable, aumentando su capacidad predictiva con el valor de su no significancia.

Tabla 6. Prueba de Hosmer y Lemeshow de los modelos de regresión logísticos.

Tabela 6. Teste de modelos de regressão logística de Hosmer e Lemeshow.

\begin{tabular}{lrll}
\hline MRL & Chi-cuadrado & gl & Sig. \\
\hline 1 & 12,025 & 8 & 0,150 \\
2 & 4,003 & 8 & 0,857 \\
3 & 2,838 & 8 & 0,944 \\
\hline \multicolumn{2}{l}{ MRL (Modelo de regresión logístico) }
\end{tabular}

MRL (Modelo de regresión logístico)

Con el objetivo de maximizar los estadígrafos de sensibilidad y especificidad se calculó el Índice de Youden (IY) utilizando cinco puntos de cortes (Pc) distintos para cada uno de los modelos de regresión logística (MRL), comenzando por 0,5 y terminando en 0,1. En la figura 2 se muestran los valores de cálculo del IY para cada uno de los modelos obtenidos, resultando tener los valores más altos los modelos dos y tres del punto de corte 0,1 . Debido a esto fue seleccionado el modelo 3 como el punto de corte óptimo para los modelos logísticos propuestos.

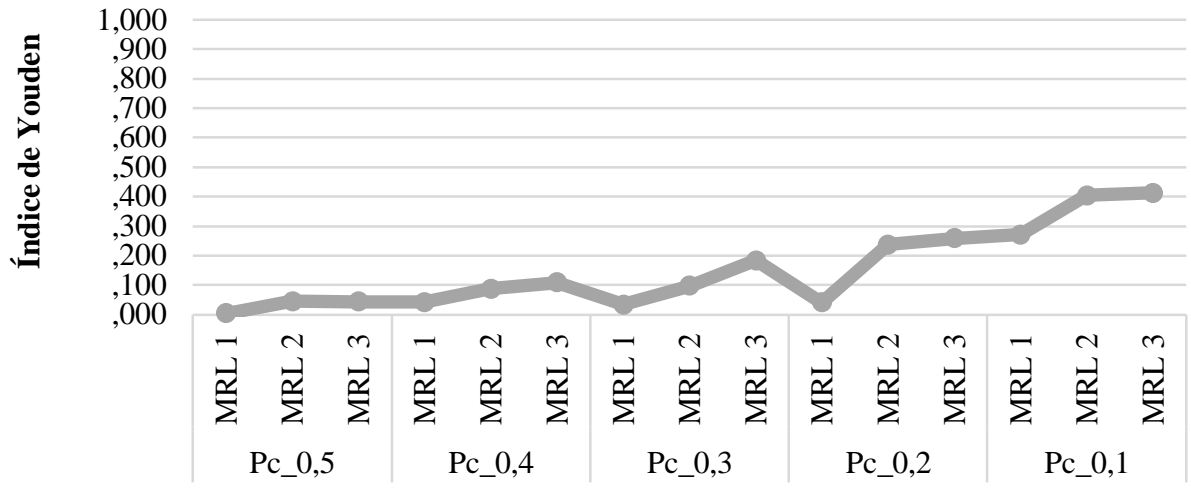

Puntos de Cortes

Figura 2. Valores del Índice de Youden en distintos puntos de cortes.

Figura 2. Valores do índice de Youden em diferentes pontos de corte.

Seleccionado 0,1 como el punto de corte óptimo que maximiza la diferencia entre las fracciones de verdaderos positivos y falsos positivos, se presenta en la tabla 7 la clasificación que hacen los modelos de los casos y la representación de la sensibilidad y especificidad para clasificarlos correctamente. La sensibilidad es el porcentaje de casos que tuvieron la característica observada, la ocurrencia de incendios, la cual fue correctamente predicha por el modelo (verdaderos positivos). En este caso, se observa el porcentaje de verdaderos positivos que fueron predichos para cada uno de los tres modelos. Según su orden, el 43,2; 79,8 y el 
$80,6 \%$ de las ocurrencias de incendios fueron correctamente predichas por el modelo. La especificidad se refiere al porcentaje de casos que no tuvieron la característica (que no ocurrieron) y fueron correctamente predichos por cada modelo de no tener esa característica (verdaderos negativos). En este caso el 83,9; 81,3 y el 82,2\% de las no ocurrencias de incendios fueron correctamente predichos por los modelos respectivamente. Al analizar el porcentaje global de ajuste determinado por cada modelo se observa que los valores son bastantes semejantes, pero si se analizan las diferencias entre los verdaderos positivos y verdaderos negativos se percibe que los modelos dos y tres presentan los menores valores con un 22 y $23 \%$ respectivamente, lo que confirma su mejora en la capacidad predictiva.

Tabla 7. Clasificación de los casos según cada modelo de regresión logística.

Tabela 7. Classificação de casos de acordo com cada modelo de regressão logística.

\begin{tabular}{|c|c|c|c|c|c|}
\hline \multicolumn{6}{|c|}{ Pronosticado } \\
\hline & \multirow{2}{*}{\multicolumn{2}{|c|}{ Observado }} & \multicolumn{2}{|c|}{ Ocurrencias de incendios } & \multirow{2}{*}{ Corrección de porcentaje } \\
\hline & & & No ocurrió & Ocurrió & \\
\hline \multirow{3}{*}{ MRL 1} & \multirow{2}{*}{ OC.Inc } & No ocurrió & 494 & 95 & 83,9 \\
\hline & & Ocurrió & 25 & 19 & 43,2 \\
\hline & \multicolumn{2}{|c|}{ Porcentaje global } & & & 81,0 \\
\hline \multirow{3}{*}{ MRL 2} & \multirow{2}{*}{ OC.Inc } & No ocurrió & 479 & 110 & 81,3 \\
\hline & & Ocurrió & 18 & 26 & 59,1 \\
\hline & \multicolumn{2}{|c|}{ Porcentaje global } & & & 79,8 \\
\hline \multirow{3}{*}{ MRL 3} & \multirow{2}{*}{ OC.Inc } & No ocurrió & 484 & 105 & 82,2 \\
\hline & & Ocurrió & 18 & 26 & 59,1 \\
\hline & \multicolumn{2}{|c|}{ Porcentaje global } & & & 80,6 \\
\hline
\end{tabular}

El valor de corte es 0,100 MRL (Modelo de regresión logística)

En la tabla 8 se presentan los valores obtenidos del Área Bajo la Curva (ABC) para cada uno de los modelos obtenidos, así como su error estándar y sus límites de confianza. Según Fuentes (2013) se suele aceptar como valor de discriminación cuando supera el valor 0,7 . Se definen entonces los modelos dos y tres como los de mayor capacidad predictiva diferenciándose solamente en $1 \%$.

Tabla 8. Área bajo las curvas COR.

Tabela 8. Área sob a curva COR.

\begin{tabular}{llllll}
\hline & & & \multicolumn{2}{c}{ Intervalo de confianza asintótico al 95 \% } \\
\hline MRL & Área & Error estándar & Sig. asintótica & Límite inferior & Límite superior \\
\hline 1 & 0,561 & 0,012 & 0,000 & 0,542 & 0,581 \\
2 & 0,707 & 0,009 & 0,000 & 0,552 & 0,582 \\
3 & 0,717 & 0,008 & 0,000 & 0,645 & 0,690 \\
\hline
\end{tabular}

Bajo el supuesto no paramétrico - Hipótesis nula: área verdadera = 0,5 MRL (Modelos de regresión logísticos)

Siguiendo las premisas planteadas por Lourenço (2006) y Preisler et al. (2008) de obtener un modelo con la menor cantidad de variables explicativas, se ha seleccionado el modelo de regresión número dos, aunque no se debe descartar la utilización del modelo número tres. La ecuación 4 representa al modelo seleccionado:

$$
\mathrm{Z}=13,608-0,276 * \mathrm{~V}-0,182 * \mathrm{Hr}
$$

Siendo la expresión de la ecuación 5, la que sustenta el diseño de la Fórmula de Pinar del Río:

$$
\mathrm{FPR}=\sum_{\mathrm{n}=1}^{\mathrm{n}} 13,608-(0,276 * \mathrm{~V})-(0,1182 * \mathrm{Hr})
$$

Dónde: FPR: Fórmula de Pinar del Río; $V$ : Velocidad del viento a las 13:00 horas; Hr: Humedad relativa a las 13:00 horas; $n$ : Número de días sin lluvias $\geq 13 \mathrm{~mm}$.

FPR es un índice acumulativo, al igual que el de Nesterov (NESTEROV, 1965), Logarítmico de Telicyn (TELECYN, 1971); Fórmula de Monte Alegre (SOARES, 1972), Índice de peligro de propagación (RODRÍGUEZ; MORETTI, 1988) y Fórmula de Monte Alegre Alterada (NUNES, 2005) expuestos por Pereira y Assunção (2008) y Secundo et al. (2015).

Para el cálculo con la FPR se utilizan tres variables: dos de forma directa como son la humedad relativa y la velocidad del viento, medidas siempre a las 13:00 horas y una de forma indirecta, que es la precipitación 
diaria acumulada en 24h. Según los criterios planteados por Turner (1970) y Soares (1972) citados por Maingi y Henry (2007), es de esperar que las variaciones en la cantidad de lluvia deben ser consideradas en cualquier índice significativo de medición del grado de severidad de la estación de incendio. Por este motivo, la precipitación, a pesar de no participar directamente en la ecuación, entra como restricción o modificación que debe ser hecha a los cálculos siempre que ocurra precipitación, de acuerdo con la tabla 2.

\section{Construcción de la escala para determinar el grado de peligro de incendios}

Para completar la estructura del índice FPR se definió su respectiva escala de peligro de incendios. En la tabla 9 se presenta la escala para la interpretación del grado de peligro de incendio estimado por el índice FPR.

Tabla 9. Escala de peligro del índice FPR.

Tabela 9. Escala de perigo para o índice.

\begin{tabular}{ll}
\hline Valor de la FPR & Grados de peligro \\
\hline$\leq 80$ & Nulo \\
$81-180$ & Pequeño \\
$181-370$ & Medio \\
$371-650$ & Alto \\
$>650$ & Muy alto \\
\hline
\end{tabular}

El comportamiento de los incendios registrados se muestra en la figura 3 demostrándose el buen ajuste y el porcentaje de variabilidad que explica una buena predicción del grado de peligro de incendios en la provincia Pinar del Río.

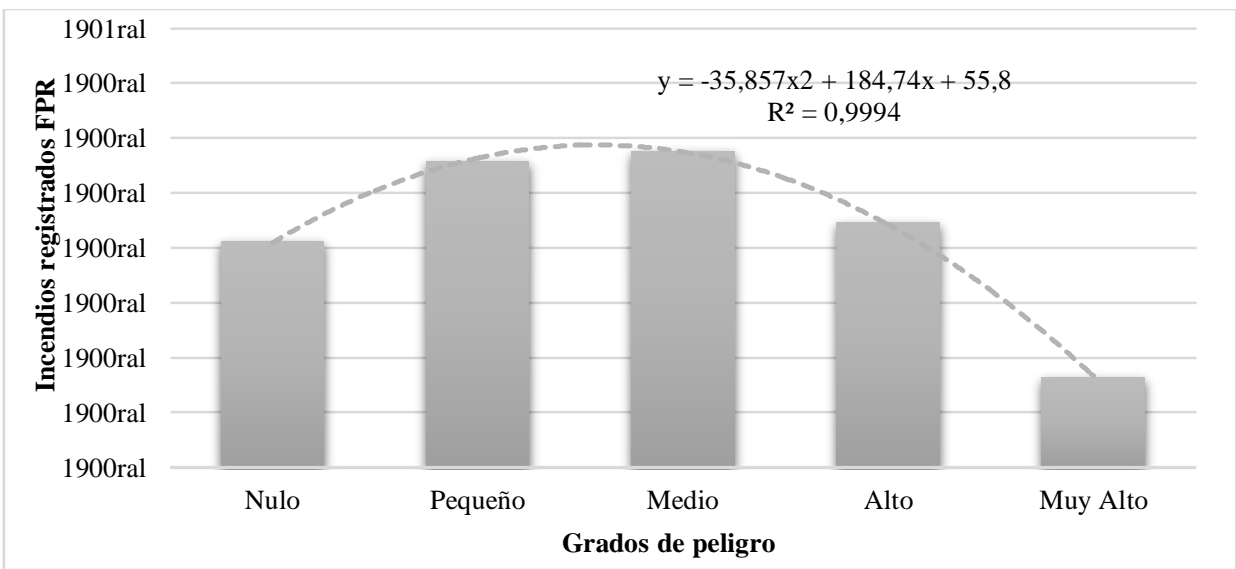

Figura 3. Número de incendios registrados en cada clase de peligro según el índice FPR.

Figura 3. Número de incêndios registrados em cada classe de perigo segundo o índice FPR.

El skill score y el valor del porcentaje de éxitos del índice diseñado fueron de 0,1174 y $63 \%$ respectivamente. Estos valores son superiores a los obtenidos por Rodríguez et al. (2012) en la EFI Macurije con la Formula de Monte Alegre Alterada con la escala ajustada, obteniendo valores de 0,0737 y 57\% para el skill score y el porcentaje de éxitos, respectivamente.

Secundo et al. (2015) expone las premisas planteadas por Nesterov (1965); Soares (1972) y Nunes (2005) los que manifiestan que un índice de inflamabilidad puede ser convertido en un índice de propagación a través de la incorporación del factor velocidad del viento. De igual forma (VASILAKOS et al., 2007; SOARES; BATISTA, 2007) plantean que un índice de peligro de incendio que incluya en su estructura la velocidad del viento permite evaluar el potencial de propagación de un incendio, factor este de gran importancia para la prevención y combate de los incendios que puedan ocurrir.

\section{CONCLUSIONES}

- Las correlaciones entre las variables meteorológicas y la ocurrencia de incendios presentaron relaciones dentro del rango moderado, bajas y muy bajas, presentándose una relación inversa entre precipitación y ocurrencia de incendios a través de los meses del año. 
- El modelo que mejor explicó la ocurrencia de incendios en la provincia Pinar del Río fue el que consideró la humedad relativa y la velocidad del viento.

- Mediante regresión logística se diseñó el modelo que mejor explica la relación entre las variables analizadas, resultando así la fórmula del índice FPR la siguiente:

$$
\mathrm{FPR}=\sum_{\mathrm{n}=1}^{\mathrm{n}} 13,608-(0,276 * \mathrm{~V})-(0,1182 * \mathrm{Hr})
$$

- El índice FPR, diseñado para la provincia Pinar del Río, refleja tanto la probabilidad de ocurrencia como la posibilidad de evaluar la propagación potencial de incendios forestales, al incluir en su estructura la variable velocidad del viento.

\section{REFERENCIAS}

CONSEJO DE DEFENSA NACIONAL (CDN). Directiva n⿳o 1 del Presidente del Consejo de Defensa Nacional para la Reducción de Desastres. Consejo de Defensa Nacional, República de Cuba. 2010. Disponible en: <http://www.sld.cu/galerias/pdf/sitios/desastres/directiva_vp_cdn_sobre_desastres.ultima_version.pdf >. Acceso el: 26/05/2016.

DOMÍNGUEZ, I. M.; MOYA, A. S.; ESTRADA, A. Vigilancia del riesgo de ocurrencia de incendios forestales mediante estaciones meteorológicas de superficie. Revista Chapingo Serie Ciencias Forestales y del Ambiente, v. 14, n. 2, p. 119-128, 2008.

FUENTES, L. E. Metodología para la elección de punto de corte óptimo para dicotomizar covariables continúas. Revista Cubana Genética Comunitaria, v. 7, n. 3, p. 36-42, 2013.

HERNÁNDEZ, C. R.; NAVARRO, R. M.; HERNÁNDEZ, B.; ESCUIN, R. S.; GARCÍA-FERRER, P. A.; SÁNCHEZ DE LA ORDEN, M. Regeneración y cambios de diversidad en grandes incendios a partir de imágenes satélite Landsat TM y ETM+. Cuadernos de investigación geográfica, v. 33, p. 85-100, 2007.

LOURENÇO, L. Incêndios florestais. Algumas reflexões sobre prevenção e mitos do combate, Territorium. Revista da Associação Portuguesa de Riscos, Prevenção e Segurança, v. 13, p. 59-70, 2006.

MAINGI, J. K.; HENRY, M. C. Factors influencing wildfire occurrence and distribution in eastern Kentucky, USA. International Journal of Wildland Fire, v. 16, p. 23-33, 2007.

MARTINEZ, J.; VEGA-GARCIA, C.; CHUVIECO, E. Human-caused wildfire risk rating for prevention planning in Spain. Journal of Environmental Management, v. 90, p. 1241-1252, 2009.

NUNES, J. R. S.; SOARES, R. V.; BATISTA, A. C. FMA ${ }^{+}$- um novo índice de perigo de incêndios florestais para o estado do Paraná, Brasil. Revista Floresta, Curitiba, v. 36, n. 1, p. 75-91, 2006.

NUNES, J. R. S.; FIER, I. S. N.; SOARES, R. V.; BATISTA, A. C. Desempenho da Fórmula de Monte Alegre (FMA) e da Fórmula de Monte Alegre Alterada $\left(\mathrm{FMA}^{+}\right)$no distrito florestal de Monte Alegre. Revista Floresta, Curitiba, v. 40, n. 2, p. 319-326, 2010.

ONEI. Anuario estadístico de Cuba. Edición 2015. La Habana, Cuba. 2014. 23 capítulos. Disponible en: <http://www.onei.cu/aec2014/02\%20Medio\%20Ambiente.pdf>. Acceso el: 28/12/2014.

ORGANIZACIÓN PARA LA AGRICULTURA Y LA ALIMENTACIÓN (FAO). Evaluación de los recursos forestales mundiales. Compendio de datos. Organización de las Naciones Unidas para la Agricultura y la Alimentación. Roma: FAO, 2015. 253 p.PEREIRA, F. T.; ASSUNÇÃO, G. Índices de risco de incêndios florestais em Juiz de Fora/MG. Revista Floresta e Ambiente, v. 15, n. 2, p. 24-34, 2008.

PREISLER, H. K.; CHEN, S. C.; FUJIOKA, F.; BENOIT, J. W.; WESTERLING, A. L. Wildland fire probabilities estimated from weather model-deduced monthly mean fire danger indices. International Journal of Wildland Fire, v. 17, p. 305-316, 2008.

RODRÍGUEZ, M. P.; SOARES, R. V.; BATISTA, A. C.; TETTO, A. F.; SIERRA, C. A.; RODRÍGUEZ, Y. C. Ajuste e desempenho dos índices de perigo de incêndios Nesterov, FMA e FMA ${ }^{+}$na empresa florestal Macurije, Cuba. Revista Floresta, Curitiba, v. 42. n. 4, p. 651-660, 2012.

SALINAS, M. Modelos de regresión y correlación III. Regresión logística. Revista Ciencia y Trabajo, v. 9, n. 24, p. 81-84, 2007.

FLORESTA, Curitiba, PR, v. 47, n. 1, p. 65- 74, jan. / mar. 2017

Rodríguez, Y. C. et al.

ISSN eletrônico 1982-4688

DOI: $10.5380 /$ rf.v47i1.47652 
SECUNDO, L. A.; ALVES, B. L.; TÂMARA, G. Evaluation of forest fire danger indexes for eucalypt plantations in Bahia, Brazil. International Journal of Forestry Research, v. 2015, p. 1-6, 2015.

SOARES, R. V.; BATISTA, A. C. Incêndios florestais: controle, efeitos e uso do fogo. Curitiba, 2007. 250 p.

SOARES, R. V.; BATISTA, A. C.; NUNES, J. R. S. Incêndios florestais no Brasil: o estado da arte. Curitiba, 2009. $246 \mathrm{p}$.

SYPHARD, A. D.; RADELOFF, V. C.; KEULER, N. S.; TAYLOR, R. S.; HAWBAKER, T. J.; STEWART, S. I.; CLAYTON, M. K. Predicting spatial patterns of fire on a southern California landscape. International Journal of Wildland Fire, v. 17, p. 602-613, 2008.

TETTO, A. F.; BATISTA, A. C.; SOARES, R. V.; NUNES, J. R. S. Comportamento e ajuste da fórmula de Monte Alegre na Floresta Nacional de Irati, Estado do Paraná. Scientia Forestalis, Piracicaba, v. 38, n. 87, p. 409-417, 2010.

VASILAKOS, C.; KALABOKIDIS, K.; HATZOPOULOS, J.; KALLOS, G.; MATSINOS, J. Integrating new methods and tools in fire danger rating. International Journal of Wildland Fire, v. 16, n. 3, p. 306-316, 2007.

VÉLEZ, R. Los índices meteorológicos de peligro. In: VÉLEZ, R. La defensa contra incendios forestales: fundamentos y experiencias. 2. ed. McGraw-Hill/ Interamericana de España, p. 151-161, 2009.

WADSWORTH, F. H. Producción forestal para América Tropical. Manual de Agricultura. Departamento de Agricultura de los EE.UU. USDA. Washington, 2000. 397 p.

YANG, J.; HE, H. S.; SHIFLEY, S. R. Spatial controls of occurrence and spread of wildfires in the Missouri Ozark Highlands. Ecological Applications, v. 18, p. 1212-1225, 2008. 